\title{
Clinicopathological Characteristics of Basal Type Breast Cancer in Triple-Negative Breast Cancer
}

\author{
Goro Kutomi $^{1}$, Tousei Ohmura ${ }^{1}$, Yasuyo Suzuki ${ }^{1}$, Hidekazu Kameshima ${ }^{1}$, Hiroaki Shima ${ }^{1}$, \\ Tomoko Takamaru $^{1}$, Fukino Satomi ${ }^{1}$, Seiko Otokozawa ${ }^{2}$, Mitsuru Mori ${ }^{2}$, Koichi Hirata ${ }^{1^{*}}$ \\ ${ }^{1}$ First Department of Surgery, School of Medicine, Sapporo Medical University, Sapporo, Japan; ${ }^{2}$ Department of Public Health, \\ School of Medicine, Sapporo Medical University, Sapporo, Japan. \\ Email: *kutomi@sapmed.ac.jp
}

Received August 28 ${ }^{\text {th }}$, 2012; revised September 25 ${ }^{\text {th }}$, 2012; accepted October $3^{\text {rd }}$, 2012

\begin{abstract}
Background: Triple-negative breast cancer (TNBC) is defined by the absence of estrogen receptor (ER), progesterone receptor (PgR) and human epidermal growth factor 2 (HER2) expression. Patients with TNBC derive no benefit from molecularly targeted treatments, such as endocrine therapy or trastuzumab, as they lack the appropriate targets for these drugs. TNBC is characterized by its biological aggressiveness and poor prognosis, and consists of two subtypes, basal and nonbasal. The purpose of our study is to differentiate the clinicopathological characteristics of the two subtypes. Methods: 367 patients with primary breast cancer were recruited from April 2004 to December 2010 at 1st Department of Surgery, Sapporo Medical University. ER, PgR, and HER2 status were evaluated in all cases. Moreover, we classified TNBC into basal, nonbasal subtypes on the basis of immunohistochemical staining of epidermal growth factor receptor (EGFR), cytokeratin (CK) 5/6. Basal type was defined as CK5/6-positive and/or EGFR-positive, and nonbasal type was defined as no expression of these two markers. Results: Breast cancer subtypes by molecular classification were Hormone receptor (HR)-positive/HER2-negative (61\%), HR-positive/HER2-positive (10\%), HR-negative/HER2positive (14\%), and HR-negative/HER2-negative (15\%). There was no difference between the basal type and the nonbasal type in clinicopathological factors. But, the basal type was significantly associated with Ki67 labeling index ( $p=$ 0.0002), p53 expression $(p=0.047)$, and BRCA1 expression $(p=0.03)$. Further, patients with the basal type TNBC showed a shorter overall survival $(p=0.032)$ than did patients with the nonbasal type. Conclusion: Classification of TNBC subtypes by EGFR, CK5/6 is a very useful prognostic factor, and highlights the need for the development of an adequate new strategy for the basal type TNBC.
\end{abstract}

Keywords: Triple-Negative Breast Cancer; Basal Type; EGFR; CK5/6

\section{Introduction}

Breast cancer is the most common female cancer. It affects more than 1 million women worldwide and about 400,000 patients die due to this disease every year. In Japan, more than 60,000 women are affected by this cancer and more than 12,000 patients die due to the disease each year. Recently, gene expression studies have identified several major subtypes of breast cancer. Triple-negative breast cancer (TNBC), characterized by the absence of estrogen receptor (ER) and, progesterone receptor (PgR) expression and no overexpression of epidermal growth factor receptor 2 (HER2), is typically associated with a poor prognosis, due to the aggressive tumor phenotype. Further, TNBC is, only partially responsive to chemotherapy and presents a lack of clinically established targeted therapies. Consequently, only con-

*Corresponding author. ventional chemotherapy is currently used in clinical practice and it effectiveness is limited [1]. Moreover, TNBC consist of two subtypes; basal and nonbasal type. The terms TNBC and basal type often are used interchangeably as there is an overlap in the biological and clinical characteristics of these tumors [2]. Basal type is similar to triple-negative breast cancer because almost all basal type do not express ER, PgR, and HER2. It has aggressive characteristics, such as high histological grade, p53 mutation, epidermal growth factor receptor (EGFR) overexpression. Some authors have claimed that the basal type is composed almost entirely of TNBC, and therefore, the TNBC phenotype could reliably be used as a surrogate for the basal type [3]. However, Rouzier [4] et al. demonstrated that ER, and HER2 expression were seen in $5 \%$ and $14 \%$, respectively, of basal type that had been diagnosed by gene expression profiling. Therefore, TNBC is not similar to basal type. In this study, we 
examined the clinical and pathological characteristics of the two TNBC subtypes. Basal type has more aggressive phenotype compared with nonbasal type. For example, basal type had higher rates of node positive than nonbasal type. In pathological perspectives, basal type was positively correlated with expression of p53, Ki67 and BRCA1.

\section{Materials and Methods}

Tissue samples were obtained from 365 patients diagnosed with breast cancer from April 2004 to December 2010 at 1st Department of Surgery, Sapporo Medical University, Japan. Other background data for the TNBC patients are shown in Table 1. The expression of ER, PgR, HER2, EGFR, CK5/6, and other biological markers was determined immunohistochemically using paraffinembedded tissue specimens. Table 2 summarizes all the antibodies, dilutions, and cutoff values used for this analysis. The expression of ER or PgR was designated as positive when at least Total score 3 on Allred score showed positive staining. Tumors that were immunohistochemically scored $3+$, or scored $2+$ and were FISHpositive, were regarded as HER2-positive. The expression of Ki67 was designated as positive when at least $20 \%$ of stained nuclei in 1,000 tumor cell nuclei [5]. The expression of CK5/6, EGFR, p53, SOX2, BRCA1 was designated as positive when at least $10 \%$ of the tumor cells showed positive staining (cytoplasmic staining for CK5/6, EGFR, SOX2, BRCA1; nuclear staining for p53) (Figure 1).

This study was approved by the ethics committee in Sapporo Medical University Hospital.

There are non-financial competing interests in this study.

\section{Statistical Analysis}

The chi-squared test and unpaired $t$-test were used for the analysis of two unpaired samples. Disease-free survival and overall survival rates after surgical resection were calculated by the Kaplan-Meier method, and differences in survival curves were assessed by the log-rank test. The Cox proportional hazards model was used for multivariate analysis. All analyses were performed with SPSS version 18.0 (SPSS Inc, Chicago IL). A $p$ value of less than 0.05 was regarded as statistically significant. All statistical tests were two- sided.

\section{Results}

Triple-negative breast cancer was diagnosed in 54 (15.3\%) of the 365 cases of operable breast cancer. The HR(+)/HER2(-), HR(+)/HER2(+), HR(-)/HER2(+), and TNBC phenotypes were identified in 224 (61\%), 36
Table 1. Clinicopathological factors in 365 primary breast cancers.

\begin{tabular}{|c|c|}
\hline Age (years), mean \pm SD & $57.4 \pm 12.5$ yesrs \\
\hline \multicolumn{2}{|l|}{ Menopause } \\
\hline Pre & $94(25.7 \%)$ \\
\hline Post & $271(74.3 \%)$ \\
\hline Tumor size $(\mathrm{cm})$, mean \pm SD & $3.0 \pm 2.6$ \\
\hline \multicolumn{2}{|l|}{ Nodal status } \\
\hline$(-)$ & $252(69.0 \%)$ \\
\hline $1-3$ & $61(16.7 \%)$ \\
\hline $4 \sim$ & $52(14.3 \%)$ \\
\hline \multicolumn{2}{|l|}{ Stage } \\
\hline I & $129(35.3 \%)$ \\
\hline II & $166(45.5 \%)$ \\
\hline III & 48 (13.2\%) \\
\hline IV & $22(6 \%)$ \\
\hline \multicolumn{2}{|l|}{ Histology } \\
\hline Pap-tub & $130(35.6 \%)$ \\
\hline Sol-tub & 39 (10.7\%) \\
\hline sci & $111(30.4 \%)$ \\
\hline Others & $85(23.4 \%)$ \\
\hline \multicolumn{2}{|l|}{ NG } \\
\hline 1 & $92(25.2 \%)$ \\
\hline 2 & $121(33.2 \%)$ \\
\hline 3 & $152(41.6 \%)$ \\
\hline \multicolumn{2}{|l|}{ ER or PgR } \\
\hline$(+)$ & $297(81.4 \%)$ \\
\hline$(-)$ & $68(18.6 \%)$ \\
\hline \multicolumn{2}{|l|}{ HER2 } \\
\hline$(+)$ & $90(24.1 \%)$ \\
\hline$(-)$ & 275 (4.5\%) \\
\hline
\end{tabular}

Pap-tub, papillotubular carcinoma; sol-tub, solid-tubular carcinoma; Sci, scirrhous carcinoma; NG, nuclear grade; ER, estrogen receptor; PgR, progesterone receptor; HER2, human epidermal growth factor receptor-2.

(10\%), 51 (14\%), 54 (15\%) of the breast cancers, respectively . TNBC was classified into two subtypes, basal (39/54, 72.2\%) and nonbasal (15/54, 27.8\%), on the basis of CK5/6 and EGFR expression. Differences in clinicopathological characteristics between the basal and nonbasal types are shown Table 3. Biological markers for the basal and nonbasal types are shown in Table 4. The basal type was positively correlated with expression of Ki67 $(p=0.0002), \mathrm{p} 53(p=0.047)$ and $\operatorname{BRCA} 1(p=$ $0.03)$. But the basal type was not correlated with 
Table 2. Antibodies, dilutions, and cutoff value.

\begin{tabular}{ccccc}
\hline Marker & Species & Manufacturer & Dilution & Cutoff value \\
\hline ER & Mouse mAb & Dako & $1: 50$ & $>$ Allred score 3 \\
PgR & Mouse mAb & Dako & $1: 100$ & $>$ Allred score 3 \\
HER2 & Rabbit poly & Dako & $1: 250$ & $3+$ or FISH+ \\
EGFR & Mouse mAb & Dako & $1: 100$ & $>10 \%$ \\
CK5/6 & Mouse mAb & Dako & $1: 100$ & $>10 \%$ \\
Ki67 & Mouse mAb & Dako & $1: 50$ & $>20 \%$ \\
p53 & Mouse mAb & Dako & $1: 50$ & $>10 \%$ \\
SOX2 & Rabbit poly & MBL & $1: 100$ & $>10 \%$ \\
BRCA1 & Rabbit poly & MBL & $1: 50$ & $>10 \%$ \\
\hline
\end{tabular}

ER, estrogen receptor; PgR, progesterone receptor; HER2, human epidermal growth receptor-2; EGFR, epidermal growth factor receptor; CK, cytokeratin; BRCA1, breast cancer susceptibility gene I; mAb, monoclonal antibody; ply, polyclonal antibody; RT, room temperature; FISH, fluorescence in situ hybridization.
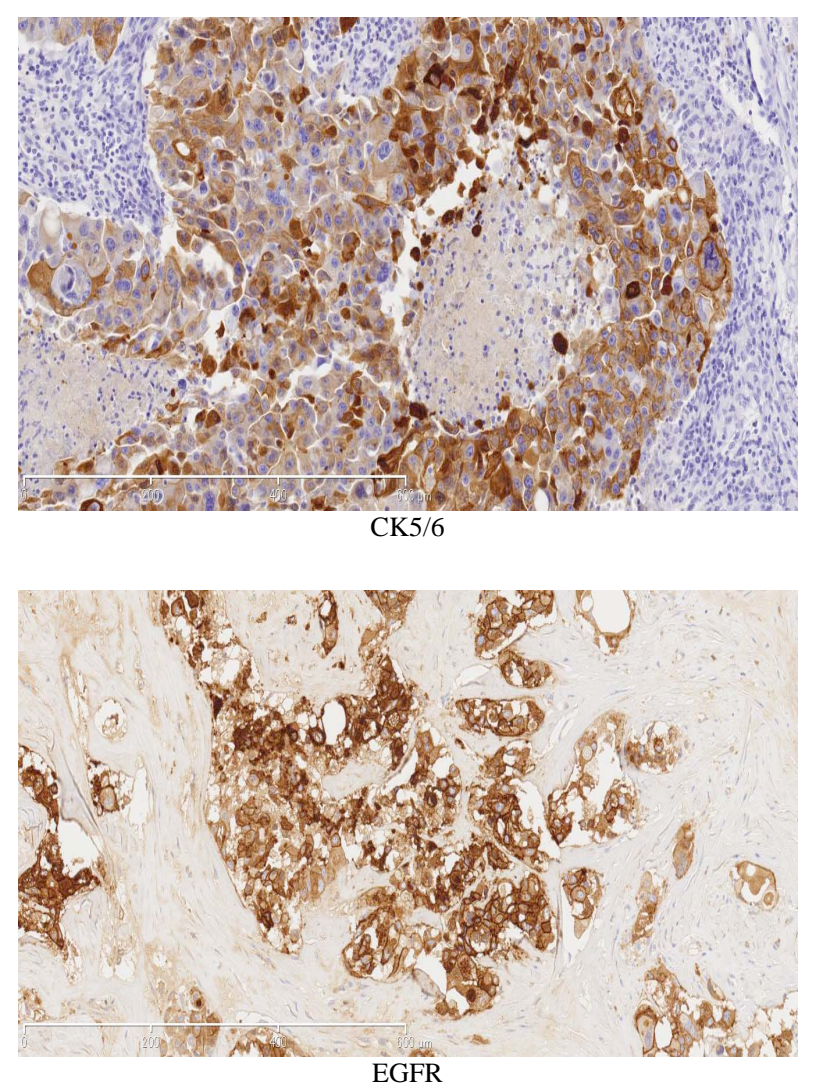

Figure 1. Breast cancer tissues were stained by CK5/6 and EGFR markers, which were mainly expressed in cytoplasmic membrane in tumor cells.

expression of $\operatorname{SOX} 2(p=0.54)$. Nonbasal TNBC is more sensitive to chemotherapy than is the basal type. So far 13 patients had been dead, in other words, the poor prognosis for basal TNBC is confirmed, which shows that $12 / 13(92.3 \%)$ of deaths were diagnosed with the basal type TNBC (data not shown).

Table 3. Clinicopathological factors according to basal subtype in triple-negative cancer.

\begin{tabular}{|c|c|c|c|}
\hline & Basal (n = 39) & Nonbasal $(\mathrm{n}=15)$ & $p$ value \\
\hline $\begin{array}{l}\text { Age (years), } \\
\text { mean } \pm \text { SD }\end{array}$ & $58.6 \pm 10.5$ & $59.0 \pm 9.6$ & 0.91 \\
\hline \multicolumn{4}{|l|}{ Menopause } \\
\hline Pre & $6(15.4)$ & $3(20.0)$ & \multirow{2}{*}{0.69} \\
\hline Post & 33 (84.6) & $12(80.0)$ & \\
\hline $\begin{array}{c}\text { Tumor size }(\mathrm{cm}) \\
\text { mean } \pm \text { SD }\end{array}$ & $3.1 \pm 2.2$ & $3.6 \pm 2.4$ & 0.48 \\
\hline \multicolumn{4}{|l|}{ Nodal status } \\
\hline$(-)$ & 21 (53.9) & $11(73.4)$ & \multirow{3}{*}{0.41} \\
\hline $1-3$ & $10(25.6)$ & $2(13.3)$ & \\
\hline $4 \sim$ & $8(20.5)$ & $2(13.3)$ & \\
\hline \multicolumn{4}{|l|}{ Stage } \\
\hline I & 5 (12.8) & $4(26.7)$ & \multirow{4}{*}{0.66} \\
\hline II & 13 (66.6) & $8(53.3)$ & \\
\hline III & $5(12.9)$ & $2(13.3)$ & \\
\hline IV & $3(7.7)$ & $1(6.7)$ & \\
\hline \multicolumn{4}{|l|}{ Histology } \\
\hline Pap-tub & $14(35.9)$ & $3(20.0)$ & \multirow{4}{*}{0.47} \\
\hline Sol-tub & $6(15.4)$ & $5(33.3)$ & \\
\hline sci & $11(28.2)$ & $4(26.7)$ & \\
\hline Others & $8(20.5)$ & $3(20.0)$ & \\
\hline \multicolumn{4}{|l|}{ NG } \\
\hline 1 & $2(5.1)$ & $1(6.7)$ & \multirow{3}{*}{0.95} \\
\hline 2 & $3(7.7)$ & $1(6.7)$ & \\
\hline 3 & $34(87.2)$ & 13 (86.6) & \\
\hline \multicolumn{4}{|l|}{ ly } \\
\hline$(+)$ & $22(56.4)$ & $8(53.3)$ & \multirow{2}{*}{0.84} \\
\hline$(-)$ & $17(43.6)$ & $7(46.7)$ & \\
\hline \multicolumn{4}{|l|}{$\mathrm{v}$} \\
\hline$(+)$ & $14(35.9)$ & $6(40.0)$ & \multirow{2}{*}{0.78} \\
\hline$(-)$ & $25(64.1)$ & $9(60.0)$ & \\
\hline
\end{tabular}

Pap-tubl papillotubular carcinoma; Sol-tubl solid-tubular carcinoma; Sci, scirrhous carcinoma; NG, nuclear grade. 
Table 4. Biological markers according to basal subtype in triple-negative cancer.

\begin{tabular}{|c|c|c|c|}
\hline & Basal (n = 39), n (\%) & Non basal ( $\mathrm{n}=15), \mathrm{n}(\%)$ & $p$ value \\
\hline \multicolumn{4}{|l|}{ p53 } \\
\hline$(+)$ & $18(47.5 \%)$ & $3(18.8 \%)$ & $p=0.047$ \\
\hline$(-)$ & 21 (52.5\%) & $12(81.2 \%)$ & \\
\hline \multicolumn{4}{|l|}{ Ki67 } \\
\hline$(+)$ & 29 (72.5\%) & $3(18.8 \%)$ & $p=0.0002$ \\
\hline$(-)$ & $10(27.5 \%)$ & $12(81.2 \%)$ & \\
\hline \multicolumn{4}{|c|}{ SOX2 } \\
\hline$(+)$ & 27 (70.0\%) & $10(62.5 \%)$ & $p=0.54$ \\
\hline$(-)$ & $12(30.0 \%)$ & $5(37.5 \%)$ & \\
\hline \multicolumn{4}{|c|}{ BRCA1 } \\
\hline$(+)$ & 35 (87.5\%) & $10(62.5 \%)$ & $p=0.03$ \\
\hline$(-)$ & $4(12.5 \%)$ & $5(37.5 \%)$ & \\
\hline
\end{tabular}

\section{Survival Analysis}

Patients with basal type TNBC had significantly shorter overall survival ( $p=0.032)$ than patients with non-basal type TNBC (Figure 2).

\section{Discussion}

Breast cancer is considered to be a heterogeneous group of tumors showing different behaviors, prognoses and responses to treatment. Although TNBCs demonstrate similarities in term of pathological, molecular and clinical characteristics, they do not represent a uniform clinical entity [6]. Despite its relatively small proportion among all breast cancers, TNBC is responsible for a large proportion of breast cancer deaths, due to its generally aggressive clinical course. But medullary and apocrine carcinomas have a better prognosis [7]. We therefore thought it important to classify basal and nonbasal types in order to resolve this discrepancy.

We demonstrated that the basal type was associated with a high Ki67 labeling index $(p=0.0002)$ and, -p53 expression ( $p=0.047)$. The high Ki67 labeling index is related to the mitotic index and high levels of cell proliferation. p53 is part of a cell cycle checkpoint, exhibiting a molecular response to DNA damage resulting in apoptosis [8]. In several studies [9-11] based on gene as well as protein expression analysis, p53 was fond to be mutated in up to $82 \%$ of basal-type TNBCs.

On the basis of a suggested link to BRCA1 [12], we investigated the expression of BRCA1. BRCA1 expression was statistically significant difference in its expression between basal and nonbasal types $(p=0.03)$.

Several studies $[13,14]$ have shown that the basal type

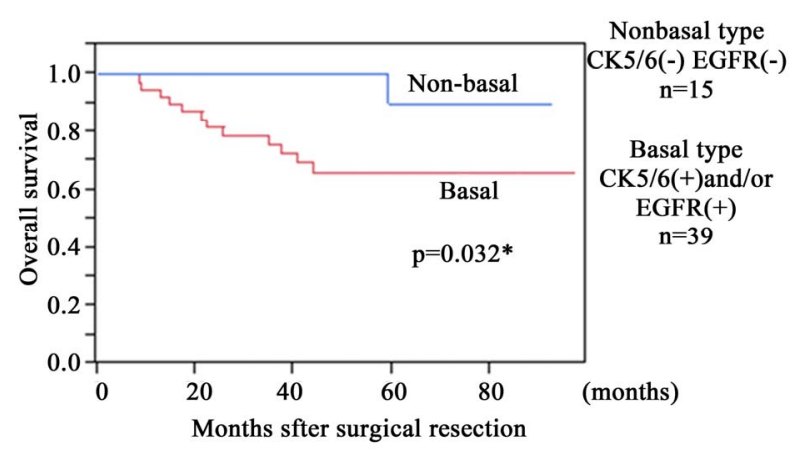

Figure 2. Overall survival according to expression of basal markers (CK5/6 and/or EGFR) in triple-negative breast cancer. There are statistically significant between basal type and nonbasal type $(p=0.032)$.

is associated with tumor size and nuclear grade; however, the difference of only nuclear grade, was observed between basal and nonbasal types in this study.

Chemotherapy remains the only systemic treatment option available for patients with TNBC. Pathological response to neoadjuvant treatment is very important because the prognosis is good for patients who achieve a pathological Complete Response, but extremely poor for those who do not. In a neoadjuvant study of, 255 patients with triple-negative disease receiving anthracycline and taxane-based regimens, Liedtke et al. [15] showed that the pathological complete response rate for TNBC was significantly higher than that identified for non TNBC (22\% versus $11 \%, p=0.034$ ). There was no significant correlation between chemotherapeutic regimen and receptor subtype. Anthracycline and taxane regimens were used as neoadjuvant chemotherapy in this study as, there were no other approved drugs. The complete response rates of the basal and nonbasal types were $12.5 \%$ and, $25 \%$, respectively, in this study (data not shown). One of the reasons is that almost advanced cases in this neoadjuvant chemotherapy. A recent study of 4000 cases compared the prognostic significance of TNBC phenotype with and without the addition of EGFR and, CK5/6. It was shown that the poor prognosis of the TNBC phenotype was conferred almost entirely by tumors positive for basal markers [16,17].

Tumors with defective DNA repair pathways, such as those displaying a BRCA1 deficiency, may be highly sensitive to DNA damaging agents such as platinumbased drugs. In a recent neoadjuvant trial of women with BRCA1 mutations and TNBC, almost $90 \%$ of patients had complete pathological responses to single agent cisplatin [18]. Results of a recent randomized phase II study of the PARP (Poly (ADP-ribose) polymerase) inhibitor BSI-201 in combination with carboplatin and gemcitabine for metastatic TNBC, showed a significantly improved clinical benefit rate and, progression-free sur- 
vival [19-21]. The development of targeted agents is, therefore, urgently needed for patients with TNBC. However, their application to routine clinical practice remains a long-term goal. In conclusion, our results indicate that is very important to classify TNBCs into basal and nonbasal types as basal- type TNBC has a significantly worse prognosis and treatment regimen must be selected accordingly.

\section{REFERENCES}

[1] F. Podo, L. Buydens, H. Degani, R. Hilhorst, E. Klipp, I. Gribbestad, et al., “Triple-Negative Breast Cancer: Present Challenges and New Perspectives,” Molecular Oncology, Vol. 4, No. 3, 2010, pp. 209-229.

[2] Y. Yamamoto, M. Ibusuki, M. Nakano, T. Kawasoe, R. Hiki and H. Iwase, "Clinical Significance of Basal-Like Subtype in Triple-Negative Breast Cancer,” Breast Cancer, Vol. 16, No. 4, 2009, pp. 260-267.

[3] T. Ruijter, J. Veeck, J. Hoon, M. Engeland and T. Tjan-Heijnen, "Characterristcs of Triple-Negative Breast Cancer," Journal of Cancer Research and Clinical Oncology, Vol. 137, No. 2, 2011, pp. 183-192.

[4] R. Rouzier, C. M. Perou, W. F. Symmans, N. Ibrahim, M. Cristofanili, K. Anderson, et al., "Breast Cancer Molecular Subtypes Respond Differently to Preoperative Chemotherapy," Clinical Cancer Research, Vol. 11, No. 16, 2005, pp. 5678-5685.

[5] J. Reis-Filho and A. Tutt, "Triple Negative Tumours: A Critical Review,” Histopathology, 52, 1, 2008, 108-118.

[6] W. Irvin, Jr. and L. Carey, "What Is Triple-Negative Breast Cancer?” European Journal of Cancer, Vol. 44, No. 18, 2008, pp. 2799-2805.

[7] M. Laurentiis, D. Cianniello, R. Caputo, B. Stanzione, G. Arpino, S. Cinieri, et al., "Treatment of Triple Negative Breast Cancer (TNBC): Current Options and Future Perspectives," Cancer Treatment Reviews, Vol. 36, Suppl. 3, 2010, pp. S80-S86.

[8] O. Gluz, C. Liedtke, N. Gottschalk, L. Pusztai, U. Nitz and N. Harbeck, "Triple-Negative Breast Cancer-Current Status and Future Directions," Annals of Surgical Oncology, Vol. 20, No. 12, 2009, pp. 1913-1927.

[9] T. Sorlie, C. M. Perou and R. Tibshirani, "Gene Expression Patterns of Breast Carcinomas Distinguish Tumor Subclasses with Clinical Implications," Proceedings of the National Academy of Sciences of the United States, Vol. 98, No. 19, 2001, pp. 10869-10874. doi:10.1073/pnas.191367098

[10] E. Korshing, J. Packeisen and K. Agelopoulos, “Cytogenetic Alterations and Cytokeratin Expression Patterns in Breast Cancer: Integrating a New Model of Breast Differentiation into Cytogenetic Pathways of Breast Carcinogenesis,” Laboratory Investigation, Vol. 82, No. 11, 2002, p. 1525 .
[11] D. Abd EI-Rehim, B. Graham and S. Pinder, "HighThroughput Protein Expression Analysis Using Tissue Microarray Technology of a Large Well-Characterised Series Identifies Biologically Distinct Classes of Breast Cancer Confirming Recent cDNA Expression Analysis," International Journal of Cancer, Vol. 116, No. 3, 2005, pp. 340-350. doi:10.1002/ijc.21004

[12] Y. Yamamoto and H. Iwase, "Clinicopathological Features and Treatment Strategy for Triple-Negative Breast Cancer,” International Journal of Clinical Oncology, Vol. 15, No. 4, 2010, pp. 341-351. doi:10.1007/s10147-010-0106-1

[13] R. Nishimura and N. Arima, "Is Triple Negative a Prognostic Facter in Breast Cancer?” Breast Cancer, Vol. 15, No. 4, 2008, pp. 303-308. doi:10.1007/s12282-008-0042-3

[14] H. Iwase, J. Kurebayashi, H. Tsuda, T. Ohta, M. Kurosumi, T. Iwase, et al., "Clinicopathological Analyses of Triple Negative Breast Cancer Using Surveillance Data from the Registration Committee of the Japanese Breast Cancer Society,” Breast Cancer, Vol. 17, No. 2, 2010, pp. 118124. doi:10.1007/s12282-009-0113-0

[15] C. Liedtke, C. Mazouni, K. Hess, F. Andre, T. Aordai, J. Mejia, et al., "Response to Neoadjuvant Therapy and Long-Term Survival in Patients with Triple-Negative Breast Cancer," Journal of Clinical Oncology, Vol. 26, No. 19, 2008, pp. 1275-1281. doi:10.1200/JCO.2007.14.4147

[16] M. C. Cheang, D. Voduc and C. Bajdik, "Basal-Like Breast Cancer Defined by Five Biomarkers Has Superior Prognostic Value than Triple-Negative Phenotype,” Clinical Cancer Research, Vol. 14, No. 5, 2008, pp. 13681376. doi:10.1158/1078-0432.CCR-07-1658

[17] S. J. Dawson, E. Provenzano and C. Caldas, "Triple Negative Breast Cancers: Clinical and Prognostic Implications,” European Journal of Cancer, Vol. 45, No. 1, 2009, pp. 27-40. doi:10.1016/S0959-8049(09)70013-9

[18] T. Byrski, J. Gronwald and T. Huzarski, "Response to Neoadjuvant Chemotherapy in Women with BRCA1Positive Breast Cancers,” Breast Cancer Research and Treatment, Vol. 108, No. 2, 2008, pp. 289-296. doi:10.1007/s10549-007-9600-1

[19] N. Turner, A. Tutt and A. Ashworth, "Targeting the DNA Repair Defect of BRCA Tumours," Current Opinion in Pharmacology, Vol. 5, No. 4, 2005, pp. 388-393. doi:10.1016/j.coph.2005.03.006

[20] H. Farmer, N. McCabe and C. J. Lord, "Targeting the DNA Repair Defect in BRCA Mutant Cells as a Therapeutic Strategy,” Nature, Vol. 434, No. 7035, 2005, pp. 917-921. doi:10.1038/nature03445

[21] P. Fong, D. Boss and T. Yap, "Inhibition of Poly (ADP-Ribose) Polymerase in Tumor from BRCA Mutation Carriers," The New England Journal of Medicine, Vol. 361, No. 2, 2009, pp. 123-134. doi:10.1056/NEJMoa0900212 